$56^{\text {ème }}$ Congrès de la SFMBCB, 02005 (2011)

DOI: $10.1051 / \mathrm{sfmbcb} / 20115602005$

(C) Owned by the authors, published by EDP Sciences, 2011

\title{
Greffes osseuses sous-sinusiennes en block par voie crestal
}

\author{
Isidori M, Fortin T, Lair JM \\ Unité fonctionnelle d'Implantologie, Hospices Civils, Lyon, France \\ isidori.michel@wanadoo.fr
}

\begin{abstract}
Objectifs : Le manque d'os dans les secteurs maxillaires postérieurs est généralement traité par l'apport d'autogreffe, d'allogreffe, de xénogreffes ou de greffons alloplastiques, à l'intérieur des sinus maxillaires, en développant une chirurgie à lambeau avec volet latéral ou bien, par manipulation de l'os résiduel à l'aide d'ostéotomes selon la technique de Summers. L'apport de la chirurgie implantaire guidée ou planifiée permet de proposer une solution alternative en développant une technique de greffe osseuse par «chirurgie minimalement invasive» afin de permettre, dans un deuxième temps, la mise en place d'implants dentaires dans les régions maxillaires postérieurs sévèrement résorbées, diminuant l'impact du geste opératoire sur nos patients.
\end{abstract}

Matériel et méthode : Sur une période de plus de 10 ans, des cylindres osseux ont été prélevés à l'aide de trépan de diamètre interne adapté au site à greffer, dans la cavité buccale ou dans des blocs d'os allogénique provenant de banque de tissus humains vivants. La longueur de chaque cylindre est définie pour être au moins égale à celle de l'implant prévu. Après abord crestal avec un geste chirurgical minimalement invasif, l'effraction de la cavité sinusienne était faite au sommet de la crête résiduelle, à l'aide de trépans de diamètre externe défini ou bien directement à l'aide d'ostéotomes. Après élévation sinusienne selon la technique de Summers, les cylindres osseux étaient ensuite introduits en force dans la cavité sinusienne par les orifices ainsi réalisés. Les sites sont ensuite refermés sans tension de manière simple. Plusieurs paramètres ont été étudiés: période de cicatrisation osseuse, gain osseux obtenu, diamètre des cylindres, diamètre et longueur des implants posés, nombre de greffes et/ou d'implants réalisés en chirurgie guidée...

Résultats : Sur une période de 10 ans, 54 greffes ont été réalisées sur 23 patients pour un gain moyen de 5,9 mm ; gains allant de 0 à $12,7 \mathrm{~mm}$. Le gain a été d'autant plus important que la hauteur d'os résiduel était faible. Il y a eu un seul échec avant la mise en place des implants. Aucune complication n'est intervenue durant la chirurgie et durant la période de surveillance, aucun implant n'a été perdu, aucune inflammation ni aucune infection n'est apparue.

Discussions : Cette étude de faisabilité suggère que l'introduction par voie crestale de cylindres osseux de dimension égale ou supérieure à celle de l'implant prévu peut être une alternative aux greffes sinusiennes avec volet osseux latéral. L'avantage de cette technique est sa rapidité de mise en œuvre, une moindre effraction du sinus et la faible quantité d'os à prélever. Ceci va dans le sens du développement des techniques de chirurgie a minima. Des études complémentaires semblent cependant nécessaires, afin d'évaluer d'autres paramètres, en particulier, l'impact médico-économique de cette technique par rapport à la technique classique. Le choix du matériau de greffe à utiliser doit se faire selon des critères à la fois médicaux, économiques, pratiques ou éthiques.

Conclusion : L'utilisation de blocs osseux cylindriques introduits par voie crestale dans le cadre du traitement des régions sous-sinusiennes radiologiquement atrophiées peut paraître, au vu de cette étude, comme une alternative au comblement de sinus. 